\title{
La guerre et le génocide de 1994: Impacts et nouveaux espoirs sur l'éducation des jeunes filles au Rwanda
}

\author{
Donatille Mujawamariya (Université d'Ottawa) \\ Susan Judith Ship
}

N'oublie pas que tu es une fille, répondit Agnès à sa fille Emma arrivée, d'urgence du Canada au Rwanda en juillet 2003, pour la consoler de la mort successive de ses deux fils et surtout la rassurer de son appui dans tous les besoins qu'elle aurait.

(Révélation d'Emma lors d'une conversation ordinaire, 2004)

Résumé: À travers un cadre théorique femme/genre et développement, les auteures mènent une discussion sur la sous-scolarisation des filles au Rwanda. Elles explorent ensuite comment les inégalités de genre interagissent avec des inégalités socioéconomiques et régionales pour entraver l'accès équitable et la participation des filles à l'éducation formelle au cours des périodes coloniale, après l'indépendance et postgénocide. Dans la suite, elles montrent comment les organisations de femmes jouent, actuellement, un rôle crucial dans la promotion de l'empowerment des filles et l'équité de genre en éducation à travers la conscientisation à la problématique de genre, des programmes et activités innovateurs.

Abstract: The authors discuss, through a women/gender and development theoretical framework, the under-schooling of girls in Rwanda. Then they explore how gender inequity interacts with socio-economic and regional inequities to establish barriers against girls in their equal access and participation to formal education during the colonial, the independence, and post-genocide periods. They show then how women's organizations currently play a crucial role in promoting women's empowerment and gender equity in education through conscientization of the gender problems, and of programmes and innovative activities.

\section{Introduction : l'équité de genre et la situation des filles en éducation au Rwanda}

Les enfants sont l'avenir du Rwanda et l'avenir des enfants au Rwanda repose en partie, sur l'importance accordée à leur éducation et leur formation professionnelle, comme le dit si bien un proverbe rwandais «kurera biruta $k u v u k a$ : éduquer c'est plus qu'enfanter » (Women's Commission for Refugee Women \& Children, 1997 :31). La guerre civile et le génocide de 1994 ont eu des effets dévastateurs sur les femmes et les enfants: on estime le nombre de veuves à 150000 , celui des enfants orphelins à 400000 et à 800000 les personnes massacrées (Women's Commission for Refugee Women \& Children, 1997:1; CISCO Learning Institute, 2000:1). Les données gouvernementales indiquaient qu'à la fin de l'an $2000,42 \%$ des femmes seraient veuves et que les femmes seraient chef de ménage dans plus de $35 \%$ de cas, la majorité vivant dans l'extrême pauvreté et sans aucun support (Newbury \& Baldwin, 2000a :6). 
En 2001, les données statistiques ont montré que $49 \%$ de la population était âgée de moins de 14 ans, $60 \%$ avait moins de 20 ans. De ces jeunes, 400000 sont des orphelins dont $20 \%$ sont chef de ménages, la majorité de ceux-là étant des filles (CISCO Learning Institute, 2000:1).

Les femmes et les filles portent la majeure partie du fardeau infligé par la guerre et le génocide de 1994 à la société rwandaise (Mukakayumba, 1995). Les horreurs dont elles ont été l'objet porte un cachet genrél comme elles ont été victimes de viol, de violence sexuelle et de traumatisme, même atteinte de VIH/SIDA, en tant que pourvoyeuses de soins aux orphelins, aux déplacés de la guerre et en tant que pilier de l'économie agricole et de la production vivrière mais aussi en assumant des nouveaux rôles politiques desquels elles étaient exclues auparavant (Newbury \& Baldwin, 2000a). Les femmes rwandaises ont mis sur pied une multitude d'associations locales, régionales et nationales qui fonctionnent en concertation grâce à un système de réseautage dans tout le pays;

Through this social organization, they have begun to rebuild their lives: reconstructing their homes, building centers for survivors of genocide and rape, learning to read and write, returning to school, acquiring new skills, participating in political life and forming local business, (Women's Commission for Refugee Women \& Children, 2001:1)

$[\grave{A}$ travers cette organisation sociale, elles ont commencé à se refaire une vie : en reconstruisant leurs maisons, en créant des centres pour les rescapés du génocide et du viol, en apprenant à lire et à écrire, en retournant à l'école, en se donnant de nouvelles habiletés, en participant à la vie politique et dans les affaires locales,] (Traduction libre).

et jouent un rôle crucial dans les efforts de reconstruction et de réconciliation de ce pays ravagé par les conflits armés.

L'empowerment de femmes comme base pour garantir l'équité de genre et un développement durable ainsi que l'égale participation des femmes comme des agents de changement sur les plans économique, social et politique (CIDA, $1997: 7)$ a été identifié comme un aspect central de la politique gouvernementale actuelle. En effet, il est appuyé par le Ministère du genre et de la promotion de la femme (MIGEPROFE) et des organismes internationaux d'aide comme UNCHR Rwanda Women's Initiative (RWI) (Women's Commission for Refugee Women \& Children, 2001; Kumar, 2001). Parmi d'importantes réalisations de RWI, on peut citer: la priorité donnée aux adolescentes habituellement marginalisées lorsqu'elles ont besoin d'assistance humanitaire et la visibilité de plus en plus croissante accordée aux problèmes relatifs au genre (Women's Commission for Refugee Women \& Children, 2001).

L'équité de genre en éducation et la situation des filles ont été définies comme éléments prioritaires devant faire partie du Plan d'action de Beijing par les Femmes Africaines dans la Déclaration de Dakar de 1994 (Labourie-Racapé, 1996) et sont aujourd'hui incorporées dans le mandat du Ministère du genre et de la promotion de la femme au Rwanda (1999) comme des secteurs prioritaires. 
Dans ce texte, nous explorons l'accès des filles à l'éducation formelle au Rwanda, dans le passé (pendant la période coloniale, après l'indépendance mais avant le génocide) et dans le contexte actuel de réconciliation et de reconstruction. Dans cette tentative d'évaluer les progrès accomplis dans la promotion et la réalisation de l'équité de genre dans l'accès à l'éducation, nous ne nous arrêterons pas seulement à l'évolution des politiques gouvernementales mais nous examinerons également le rôle crucial joué par des organisations de femmes afin de promouvoir l'empowerment des filles et des femmes ainsi que l'atteinte de l'équité de genre en matière d'éducation des filles.

La situation de l'éducation des filles au Rwanda a beaucoup de points en commun avec la sous scolarisation des filles de plusieurs autres pays africains et est étroitement liée à l'existence des inégalités de genre, au détriment des filles et femmes. Entre autres ces inégalités, il convient de mentionner d'une part des inégalités socio-économiques qui favorisent des élites économiques et d'autre part des inégalités régionales qui privilégient des milieux urbains. Ces inégalités sont l'héritage des systèmes d'éducation mis sur pied pendant la période coloniale (Jefremovas, 1992; Cocquery-Vidrovitch, 1994; Okojie, 2001). Toutefois, le Rwanda fait face à des défis uniques en matière d'équité de genre en éducation suite aux conséquences de la guerre et du génocide de 1994. Entre autres ces défis figurent la reconstruction de l'infrastructure scolaire, la reconstitution d'un corps enseignant, les nombreux orphelins et enfants chefs de ménage, surtout les filles, victimes de viol et de VIH/SIDA, qui ont des besoins spécifiques dont la guérison, l'assistance psychosociale et d'autres formes de support pour leur survie (Women's Commission for Refugee Women \& Children, 2000; Newbury and Baldwin, 2000a). Malgré les efforts du gouvernement pour améliorer l'accès des filles à l'éducation, la parité ne pourra être atteinte sans qu'il y ait une transformation à la fois des inégalités de genre et des autres inégalités structurelles qui entravent l'entière et équitable participation des filles en éducation. Les organisations de femmes au Rwanda jouent un rôle fondamental en agissant comme des agents de changement dans l'empowerment des filles et l'équité de genre en éducation en plus de pourvoir aux besoins de plusieurs orphelins.

Ce texte commence par une discussion sur les causes de la sousscolarisation des filles au Rwanda et appuie la nécessité de recourir à un cadre théorique femme/genre et développement. Nous explorons ensuite comment les inégalités de genre interagissent avec des inégalités socio-économiques et régionales pour contrer l'accès équitable et la participation des filles à l'éducation formelle au cours des périodes coloniale, après l'indépendance et post-génocide. Dans la suite, nous montrons comment les organisations de femmes jouent un rôle essentiel dans la promotion de l'empowerment des filles et l'équité de genre en éducation à travers la conscientisation et des programmes et activités innovateurs. 


\section{Femme/genre et développement et l'éducation des filles au Rwanda}

Explorer la question de l'accès des filles à l'éducation au Rwanda hier et aujourd'hui nécessite que nous nous inscrivions dans une perspective théorique femme/genre et développement (Rathgeber, 1994) qui conçoit le genre en tant que rapports sociaux d'inégalité et dans son interaction avec d'autres rapports sociaux tels que la classe sociale qui façonnent la situation de subordination des femmes. Il serait en effet difficile de comprendre les inégalités de genre en éducation au détriment des filles sans, d'une part, identifier les contraintes auxquelles les filles et femmes font face suite à leur triple rôle; dans la reproduction (travaux domestiques et soins aux enfants), dans la production (agricole et économie informelle), et dans le service à la communauté (bénévolat) et d'autre part, connaître leurs besoins et opinions en matière d'éducation. L'attitude continuellement répandue selon laquelle les rôles sociaux des femmes dans la reproduction et la procréation en tant mères et épouses sont d'une importance capitale, à laquelle s'ajoute une série de traditions et coutumes qui privilégient les hommes, ont contribué à limiter la participation des femmes rwandaises dans des sphères publiques, les reléguant ainsi à un statut subordonné comme des citoyens de deuxième classe. Les rôles traditionnels et les inégalités de genre sont à l'origine de l'accès limité des femmes et des filles à l'éducation formelle ainsi qu'à la formation professionnelle et à d'autres formes d'éducation non-formelle. De plus, les inégalités de genre dans l'accès à l'éducation au Rwanda sont intensifiées d'une part par des disparités régionales qui favorisent les zones urbaines par rapport aux zones rurales et d'autre part par la structure et le coût de l'éducation qui privilégient les plus favorisés au détriment de la vaste majorité des Rwandais, économiquement défavorisée, qui vit dans la pauvreté (World Bank, 2003). Comme Gatali (2000:51) le souligne dans son étude sur la sous-scolarisation et la déperdition des filles:

La faible scolarisation des filles est à la fois liée à l'héritage de l'enseignement colonial, aux problèmes propres à la mentalité de la population rwandaise dont l'idéologie dominante est l'hiérarchisation des sexes, qui accorde peu de crédit aux capacités de la femme et à l'égalité des sexes, aux problèmes économiques, qui font que les familles confrontées à la pauvreté consacrent les ressources disponibles aux études des garçons, puisque appartenant à la famille alors que la fille est destinée à être ailleurs. Dans ce contexte, le travail de la fille est considéré non seulement comme un appui économique à la famille, mais aussi comme un élément de formation traditionnelle, exercice indispensable à la préparation à son statut de future épouse et de gestionnaire du foyer. Le handicap subi par la fille rwandaise dans sa scolarisation étant à la fois d'ordre culturel et économique, une action vigoureuse, faite de mesures politiques et de changement de mentalité doit être menée par les instances chargées de la promotion de la femme. Contrairement aux propos de Gatali, notre étude ne se limite pas uniquement au rôle du Ministère du Genre et de la Promotion de la Femme dans la 
conscientisation des femmes à la question de genre et équité en matière d'éducation des filles. Nous explorons aussi le rôle crucial des organisations des femmes dans l'empowerment des femmes et des filles et la lutte pour l'équité de genre en éducation.

Notre étude repose sur un modèle de recherche participative communautaire qui met de l'avant une conception d'empowerment (Ristock and Pennell, 1996). Cette méthodologie vise à garantir l'accès, le contrôle et l'appropriation de la recherche et de son objet par les organisations de femmes rwandaises. Elle se marie bien à une approche femme/genre et développement pour l'éducation des filles (Rathgeber. 1994). Selon Kumar (2001:31):

Empowerment refers to an individual or a group's ability to influence matters affecting its welfare and interests. In the context of gender relations, empowerment connotes that women have the freedom to decide for themselves. They interact with men from a position of equality rather than from perpetual disadvantage. Thus women are empowered when social, cultural, economic or political barriers are removed or eliminated and they can realize their full potential.

[L'empowerment réfère à l'habilité qu'a une personne ou un groupe d'influencer ce qui affecte son bien-être ou ses intérêts. En ce qui a trait aux rapports de genre, l'Empowerment signifie que les femmes ont la liberté de décider pour elles-mêmes. Elles interagissent avec les hommes d'égal à égal. L'empowerment des femmes implique que des des barrières sociales, culturelles, économiques et politiques soient éliminées afin que les femmes puissent se réaliser à leur plein potentiel.] (Traduction libre).

Et comme Dagenais (1994:263) nous le rappelle: "le processus d'empowerment comporte deux dimensions, une personnelle et une collective, qui se renforcent mutuellement et sans lesquelles il ne peut véritablement en être question." L'empowerment comme approche de recherche communautaire nécessite une prise de conscience des relations de pouvoir, du contexte culturel et de l'action sociale (Ristock et Pennell, 1996: 2). En appliquant cette notion à une recherche avec des femmes au Rwanda, l'approche permet de construire un savoir qui vise à changer les conditions de vie des femmes individuellement et collectivement. Cela implique la consultation et la collaboration avec divers regroupements de femmes comme partie intégrante du processus d'amélioration des conditions de vie des femmes.

Fondamentalement, c'est une recherche qui permet d'identifier, de faciliter ou de créer des contextes dans lesquels des groupes qui n'ont pas droit à la parole, comme ce fut le cas des femmes au Rwanda, mis à l'écart du processus décisionnel dans tous les aspects de la vie, acquièrent le savoir, ont une voix et une influence dans les décisions qui concernent leur existence. Afin de nous assurer que nos résultats reflètent véritablement les besoins, expériences et perspectives des femmes dont elles se serviront ensuite dans leurs efforts pour l'équité de genre et un développement durable, nous avons utilisé cette méthodologie pour mener des entrevues auprès des responsables des 
organisations de femmes (Réseau de Femmes oeuvrant pour le développement rural-Gitarama; Pro-femmes/Twese Hamwe Kigali/Gitarama), des organisations de femmes à caractère religieux (Mamans catholiques/Gitarama; Caritas/Diocèse de Kabgayi), le Ministère du genre et de la promotion de la femme mais aussi des organisations internationales et organismes d'aide comme Unifem; PNUD; UNICEF; Banque Mondiale et SNV/Pradec.

\section{L'héritage colonial : les filles moins scolarisées que les garçons}

Ce n'est qu'au début des années 1900 que l'école moderne voit le jour au Rwanda avec l'avènement du colonialisme et de l'évangélisation. En effet, l'école telle qu'on la connaît aujourd'hui est apparue au Rwanda entre 1900 et 1906 alors que la première école ouvre ses portes au Sud du pays plus précisément à Save. Par leur installation physique, les missionnaires modernisaient leur zone et ses environs créant du même coup des disparités avec des zones éloignées. Ils sont à l'origine de la division du pays en zones urbaines et rurales, les écoles étant bien sûr situées dans les zones urbaines.

Il importe cependant de souligner qu'il était hors question, à cette époque, d'envoyer les filles à l'école. L'évangélisation n'étant pas bien accueillie par le peuple rwandais qui n'était pas prêt à abandonner ses coutumes, ses valeurs et sa culture à la merci d'une civilisation européenne que les missionnaires voulaient introduire, les parents n'envoyaient à l'école que les enfants en situation déplorable. Les filles étaient protégées par la famille et ne devaient donc pas s'en écarter (Berger, 1990; UNR, 1978). Néanmoins, l'ouverture des écoles primaires pour garçons s'est poursuivie au fur et à mesure que les missions étaient implantées sur le territoire rwandais (Gatali, 2000). Toutefois, il faudra attendre l'arrivée des sœurs blanches pour que s'ouvre la première école pour les filles, également à Save et dont l'objectif se limitait à préparer les futures religieuses rwandaises. En 1910, le Rwanda comptait 33 écoles primaires avec 1250 élèves, tandis qu'en 1915 il y avait 40 écoles abritant 2000 élèves dont 500 filles (Paternostre, 1983). Sous mandat belge, l'école primaire a connu une expansion. De 1949 à 1960 , le nombre d'élèves a plus que doublé passant de 112000 à 265000 dont $31 \%$ de filles (Paternostre, 1983).

Bien avant 1930, les garçons pouvaient déjà fréquenter des écoles secondaires comme l'École de Moniteurs de Save, le Petit Séminaire de Kabgayi, et le Groupe Scolaire de Butare. La première école post-primaire pour les filles n'ouvrira les portes qu'en 1939 dans les bâtiments de l'École des Moniteurs de Save celle-ci déménagée à l'Est du pays, à Zaza. La mission de cette école ménagère qui durait deux ans était double; préparer les futures religieuses, et former les futures épouses de la nouvelle élite dirigeante sortie des établissements secondaires: ainsi fut introduite la domestification de la femme, valeur importée du système patriarcal occidental (Djossou-Segla et Mujawamariya, 1998; Mujawamariya, 1992). Il faut noter cependant que les filles qui se destinaient à la religion bénéficiaient d'une année pédagogique supplémentaire les préparant de ce fait à l'enseignement. La mixité étant interdite à cette époque, très peu d'écoles primaires pouvaient être ouvertes à 
cause du nombre limité d'enseignantes (les religieuses) (Gatali, 2000). Ce qui par voie de conséquence affecte dramatiquement la scolarisation de jeunes filles.

À partir des années 1952, deux écoles normales moyennes accessibles aux lauréates laïques sont ouvertes (Byimana, Rwaza). Et vers la fin des années 1950 , deux écoles infirmières, réservées uniquement aux filles, sont ouvertes (à Kabgayi et à Rwamagana). Il va sans dire que la scolarisation des filles a connu un sérieux retard pendant la période coloniale. Les écoles primaires pour filles sont très peu nombreuses car l'ouverture de ces dernières était conditionnelle à la disponibilité du corps enseignant féminin, or, les premières écoles de formation d'enseignantes laïques ayant été ouvertes en 1952. Pendant que les garçons pouvaient bénéficier d'une formation dans des écoles diversifiées (normales, administratives, agricoles, médicales, vétérinaires, séminaires...), les filles ne pouvaient se contenter que d'une formation soit dans des écoles normales ou infirmières (Gatali, 2000 :2). À la fin de l'époque coloniale, non seulement les filles sont moins scolarisées que les garçons, mais aussi elles ont été confinées dans des disciplines traditionnellement féminins.

\section{De l'indépendance à 1994 : accession tardive et lente des filles à l'enseignement supérieur}

Au cours des premières années de l'indépendance politique conquise en 1962, le sort réservé aux filles était quasiment le même que pendant l'époque coloniale. Toutefois, la constitution de 1962 en rendant l'école primaire obligatoire et gratuite $^{2}$ a permis que les effectifs des filles se rapprochent de ceux des garçons. L'école primaire connaîtra cependant un problème de sélection qui se fait à la fin de ce programme pour passer au secondaire. Le processus étant très sélectif sur base des résultats scolaires, la majorité des élèves connaissent déjà leur destin : le retour au village pour travailler au champ. Les parents préfèrent ainsi récupérer leurs enfants ou ces derniers décident eux-mêmes d'abandonner l'école, les filles en premier car la famille a besoin de leur aide pour les travaux domestiques (Uwimana, $1992: 18$ ).

Néanmoins, la réforme de 1963-1964 a marqué un tournant dans la scolarisation des filles et garçons: les garçons qui ne réussissent pas l'examen ministériel pour entrer à l'école secondaire sont envoyés dans des Centres d'enseignement rural et artisanal (CERAR) alors que les filles sont dirigées dans des sections familiales (SF). Bien qu'elles aient la possibilité de poursuivre les études, la section familiale que ces filles fréquentent prépare de futures ménagères (cuisine, couture, broderie, puériculture...) candidates au mariage avec la nouvelle élite de l'époque, tout comme à l'époque coloniale. Toutefois, la mixité à l'école primaire est permise, ce qui aura pour effet d'accroître le nombre de filles à l'école primaire.

Cependant, la proportion des filles par rapport aux garçons diminue au fur et à mesure qu'on monte dans les niveaux d'enseignement. En 1987, 24\% des élèves étaient des filles en première année tandis qu'elles n'étaient que de $6 \%$ en dernière année de l'école primaire (Uwimana, $1992: 17$ ). L'étude de Gatali $(2000: 14-15)$ révèle que pour l'année 1990-1991, les filles ont plus de 
succès au niveau du $1^{\text {er }}$ cycle de l'enseignement primaire (les trois premières années) avec $2,1 \%$ de plus que les garçons alors que c'est l'inverse au 2ème cycle (les trois dernières années) avec plus de $3,1 \%$ en faveur des garçons. Une analyse du taux de redoublement, pour la même année, amène ce dernier auteur à conclure que les garçons sont encouragés à rester à l'école plus longtemps que leurs sœurs, ce qui leur donne ainsi la chance de passer éventuellement au secondaire.

La sélection pour le secondaire touche donc les filles avec plus d'acuité que les garçons et leur nombre diminue au fur et à mesure qu'on avance dans les années d'études. Pendant qu'elles ne représentaient que $30 \%$ en 1987 en première année du secondaire, elles ne sont que de $8 \%$ en dernière année (sixième) (Uwimana, $1992: 19$ ). Tout comme au primaire, au secondaire elles ont moins de chance de redoubler l'année que leurs frères. En 1985, sur un nombre total de 276 redoublants 48 étaient des filles. En 1986, sur 319, seulement 4 filles ont bénéficié du redoublement (Uwimana, 1992: 25).

Ce n'est qu'en 1968 que la première école de sciences pour les filles voit le jour au Rwanda, à Nyundo. Néanmoins, la réforme de 1980 aura un impact remarquable sur le nombre des filles au secondaire grâce à deux mesures introduites par le gouvernement d'alors; la mixité au secondaire est institutionnalisée et le principe d'externat est accepté permettant ainsi de libérer des places et d'accroître le nombre total des effectifs d'élèves. D'après les données statistiques disponibles (Gatali, 2000), des élèves qui fréquentaient l'école secondaire en $1980,23,3 \%$ étaient des filles comparativement à $38,9 \%$ en 1990-1991, ce qui est quand même une augmentation remarquable (167\%). Il est regrettable, cependant, de constater que l'Église catholique, qui en ce moment gérait plus de $75 \%$ des écoles, était réticente à la mixité, ce qui réduisait de ce fait la mise en application de cette mesure. Rappelons ici que l'enseignement est dès ses premiers jours une cuvre des missionnaires catholiques. Comme les niveaux d'enseignement sont interdépendants, le primaire préparant le secondaire, ce dernier préparant le supérieur, les problèmes relevés ci-haut au secondaire ont un impact direct sur le supérieur.

L'enseignement supérieur ne verra le jour qu'en 1963 avec la création de l'Université Nationale du Rwanda (UNR) suivie en 1966 par l'Institut Pédagogique National (IPN). Depuis l'avènement de l'école au Rwanda, l'enseignement supérieur était poursuivi à l'étranger jusqu'à cette période. Pour accéder à l'enseignement supérieur, il fallait avoir complété avec succès les humanités, ce qui n'était pas le cas pour la plupart des filles car l'école ne les y avait pas préparées, à l'exception faite pour celles qui avaient fréquenté l'école des sciences de Nyundo qui n'a vu le jour, comme mentionné plus haut, qu'en 1968.

L'année internationale de la femme (1975) constituera un tournant majeur car les quelques rares femmes ayant fréquenté l'école supérieure revendiquent une plus grande accessibilité des femmes à l'enseignement supérieur. En 1980, le pourcentage des filles fréquentant les établissements d'enseignement supérieur s'élevait à $8,2 \%$. Dix ans plus tard, c'est-à-dire en 
1990-1991, ce chiffre avait plus que doublé passant de $8,2 \%$ à $21,5 \%$ (Gatali, 2000, Uwimana, 1992). Mais toujours est-il que jusqu'en 1994, les filles sont très peu nombreuses dans l'enseignement supérieur comparativement aux garçons. Et le système a toujours privilégié les milieux urbains au détriment des zones rurales ainsi que les étudiants des classes socio-économiquement favorisées au détriment de ceux provenant de familles moins nanties. Comme on va le voir dans la section suivante, avec la guerre et le génocide de 1994, ces tendances se sont maintenues même qu'elles se sont accentuées pour ce qui est des filles.

\section{De 1994 à aujourd'hui : Qu'y a-t-il de changé au cours de la décennie ?}

La guerre et le génocide de 1994 ont eu des effets dévastateurs sur le système éducatif rwandais: on estime à 600 écoles primaires soit $32 \%$ du total détruites, 3000 instituteurs tués, des milliers des enfants qui ont interrompu leurs études et même l'Université nationale avait dû fermer ses portes pour ne rouvrir qu'en 1995 (CISCO Learning Institute, 2002 :2; Gouvernement de la République Rwandaise et UNICEF, $2000: 86$ ). Par conséquent, les quelques écoles qui existent sont surchargées et les enseignants non qualifiés: en 1998/1999, au primaire, le rapport élèves/enseignant est de 59 et le taux de qualification des enseignants s'élève uniquement à $49,2 \%$ (Gouvernement de la République Rwandaise et UNICEF, 2000). En 2000, le rapport élèves/enseignant est passé à 52 , mais la pénurie des enseignants, particulièrement au secondaire et au supérieur se faisait toujours sentir conjuguée à des mauvais salaires, au manque de formation et à une carence de fournitures (Nationmaster.com, 2004: 2; Canadian International Development Agency, 2002:3). Néanmoins, une étude récente de la Banque mondiale au Rwanda (World Bank, 2003: xv) indique que des écoles ont été réparées, d'autres ont été construites, d'anciens maîtres ont été réintégrés, le nombre d'élèves a augmenté, les arriérés salariaux ont été payés, un fonds Génocide a été créé pour venir en aide aux orphelins, l'enseignement secondaire et supérieur est plus diversifié avec l'intensification du privé, des arrangements ont été conclus afin de permettre aux étudiants du supérieur de payer leurs études. Bref, il y a de quoi être optimiste. Ses succès sont en partie le fruit des efforts du gouvernement actuel qui s'est beaucoup investi dans la reconstruction du système d'éducation afin de démocratiser l'enseignement primaire.

La Banque Mondiale (World Bank, 2003: 3) signalait que only five years after the genocide, the number of children in primary school surpassed the number that would have been enrolled had the system expanded at historical rates of increase and the current gross enrollment ratio of $107 \%$ exceeds the corresponding ratio for the average low-income country in Africa today.

[seulement cinq ans après le génocide, le nombre des élèves à l'école primaire a dépassé le nombre auquel on pourrait s'attendre pour atteindre un taux d'inscription de $107 \%$ qui est supérieur au taux 
moyen des inscriptions dans les pays à faible revenu en Afrique.] (Traduction libre).

Bien que des mesures aient été mises en place pour s'assurer qu'une forte proportion des orphelins (particulièrement ceux qui ont perdu un parent) aient accès à l'école primaire, il persiste un écart remarquable parmi les enfants les plus vulnérables qui fréquentent l'école: les double-orphelins et les enfants qui vivent séparés de leurs parents (World Bank, 2003 :3). Les inégalités de genre à l'accès à l'éducation sont plus prononcées chez les filles orphelines. Siaens et al. (2002) faisaient remarquer que chez les orphelins le taux de scolarisation était plus faible alors qu'ils avaient le taux le plus élevé en matière du travail des enfants, ce phénomène étant plus accentué chez les filles que chez les garçons. Le placement dans des familles d'accueil n'a guère amélioré la situation de ces filles orphelines, pour leur permettre d'aller à l'école, car elles sont toujours sollicitées à participer aux travaux domestiques.

Malgré le taux de fréquentation élevée des enfants rwandais à l'école primaire ( $88 \%$ de tous les enfants), le taux de rétention reste en général très faible; seulement $39,1 \%$ de ces enfants ont réussi à se rendre jusqu'en cinquième du primaire, entre 1998 et 2000 (Nationmaster.com, 2004 :2). Les inégalités de genre et socio-économiques ont beaucoup d'impacts sur les taux de rétention à l'école. Malgré que les barrières institutionnelles ont été légalement éliminées et qu'aujourd'hui il y a parité de genre au primaire--le rapport net des filles en 1998-99 était de 97 et le taux net de scolarisation au primaire (\%femmes/garçons) était de 1.0 pour la même année (PNUD, 2003 :9)--le taux d'abandon des filles reste plus élevé que celui des garçons (Hamilton, 2000: 6; Gouvernement de la République Rwandaise et UNICEF, $2000: 81$ ). De plus, malgré le taux d'inscription élevé des filles,

in practice, they attend less, achieve less and drop out much more than boys do, often as a result of pregnancy. Reproductive health education is nominally provided but more can be done in this area. (Women's Commission for Refugee Women and Children, 2000:12).

[en pratique, elles se présentent moins au cours, réussissent moins et abandonnent l'école beaucoup plus que les garçons, souvent à cause des grossesses. En principe, une éducation sur la santé reproductive est offerte mais il y a encore beaucoup à faire dans ce domaine.] (Traduction libre)

L'UNICEF (1997:108) a, pour sa part, souligné que

the disparity is often the result of survival strategies of poor families, who withdraw their female children first if there is not enough money to pay for the various costs associated with schooling.

[l'écart résulte des stratégies de survie dont usent les familles pauvres consistant à retirer en premier temps leurs filles de l'école lorsqu'elles ne disposent pas de moyens financiers suffisants pour couvrir les dépenses reliées à la scolarisation.] (Traduction libre).

Comme l'éducation n'est pas gratuite au Rwanda et entraîne d'autres coûts substantiels comme l'achat des uniformes et des livres, les familles pauvres 
doivent faire un choix difficile sur le nombre d'enfants qu'ils peuvent financièrement envoyer à l'école (Hamilton, 2000). Les filles particulièrement celles des zones rurales ${ }^{3}$ sont surreprésentées parmi ceux qui n'arrivent pas à compléter l'école primaire.

The 1996 Socio-Demographic Study carried out by the government found that, unsurprisingly for a developing country, $24 \%$ of children from ages 10 to 14 are economically active. The report pointed out, however, that the proportion of girls in this group was higher than expected and the majority works in the agricultural sector (République du Rwanda, 1998:25).

[L'étude socio-démographique conduite en 1996 par le Gouvernement rwandais a révélé, curieusement pour un pays en voie de développement, que $24 \%$ des enfants âgés de 10 à 14 ans sont économiquement actifs. Par ailleurs, cette étude fait remarquer que dans ce groupe, le nombre de filles est plus élévé que celui des garçons et que la majorité d'elles s'adonnent à des activités du secteur agricole.] (Traduction libre).

De plus, pour les filles qui sont chefs de ménage, le temps et l'argent pour aller à l'école ou suivre une formation est une réalité inenvisageable.

Les disparités de genre, géographiques et socio-économiques sont les plus prononcées aux niveaux supérieurs d'éducation, à l'école secondaire et aux études post-secondaires (World Bank, $2003: 3$ ). Au niveau secondaire, le nombre d'étudiants a maintenant augmenté de $20 \%$ par an depuis 1996 , ce qui laisse supposer que le système est presque trois fois plus large qu'il ne l'était avant.Toujours selon la Banque Mondiale (World Bank, 2003: 4),

in secondary education orphans are at least as well represented as nonorphans. This remarkable outcome owes much to the government's decision in 1998 to establish the tax-funded Genocide Fund as a mechanism for assisting orphans in secondary school .

[au secondaire, on retrouve autant des enfants orphelins que ceux qui ne le sont pas. On doit ce succès à la décision du Gouvernement rwandais de créer depuis 1998 un Fonds d'aide aux rescapés du génocide pour porter assistance aux orphelins dans l'enseignement secondaire.] (Traduction libre).

Cependant, le taux de scolarisation au secondaire est toujours faible et des disparités de genre persistent. De 1996 à 1998, seulement 14,9\% de garçons et $11,5 \%$ de filles fréquentaient l'école secondaire (Banque Africaine de Développement, 2002-2003:29). Il est important de noter que pendant la période post-conflit,

the share of students attending private schools remained steady at $40 \%$ down from $62 \%$ in 1980 s-but still much higher than the average $20 \%$ for low-income sub-Saharan Africa » (World Bank, 2003: 3).

[la part des étudiants fréquentant le privé est resté à $40 \%$-inférieur à celle des années 1980 qui s'élevait à $62 \%$ - mais toujours supérieur à la 
moyenne de $20 \%$ des pays à faible revenu de l'Afrique Subsaharienne.]

(Traduction libre).

Par conséquent, la plupart des étudiants qui fréquentent l'école secondaire proviennent des classes socio-économiques favorisées.

La plupart des écoles secondaires ne disposent pas d'infrastructures d'accueil pour les filles. Par conséquent, lorsqu'elles fréquentent des écoles mixtes, ces filles doivent se débrouiller pour trouver une chambre à louer ou rester chez des proches ou simplement partager un logement avec des étrangers, "and they are said to be badly regarded and treated as servants" (Women's Commission for Refugee Women and Children, 2000: 12) [et on dit d'elles qu'elles sont mal regardées et traitées comme des servantes.] (Traduction libre). Seulement un petit nombre de filles qui vont à l'école secondaire arrivent à compléter leurs études et le taux élevé d'abandon de filles à l'école secondaire reste un sujet de préoccupation majeure. Comme le soulignent Newbury et Baldwin (2000a:9-10):

Of primary students who took the final exam in 1995-1996, only 16 percent qualified for admittance to a state or state-subsidized school. Another 10 percent were able to attend private secondary schools at great cost to their families...Many young women saw their education interrupted by the war and the genocide. Those who had reached secondary school in particularly are positioned to benefit enormously from an opportunity to continue studies. Adult education programs enabling these women to study would build on the education they have already acquired and provide them with the skills they desperately need.

[Des élèves qui ont pris part aux examens de fin d'études primaires de 1995-1996, seulement 16\% ont été admis dans une école secondaire publique ou subsidiée. L'autre $10 \%$ ont pu poursuivre leurs études secondaires dans des écoles privées aux frais de leurs familles. Beaucoup de jeunes filles ont vu leurs études interrompues à cause de la guerre et du génocide. Celles qui ont pu arriver au secondaire ont la chance de pouvoir poursuivre leurs études. Les programmes d'éducation des adultes qui permettent à ces filles et femmes de compléter leurs études devraient construire sur le bagage qu'elles ont déjà et leur permettre d'acquérir des habiletés dont elles ont le plus besoin.] (Traduction libre).

Pour les années 1998 et 1999 , des filles qui s'inscrivaient aux examens de fin d'études secondaires, $82 \%$ et $66 \%$ ont réussi à obtenir leur diplôme comparativement à $88 \%$ et $76 \%$ chez les garçons (Gatali, $2000: 21$ ). Il s'avère par ailleurs que lorsqu'elles fréquentent l'école secondaire ces filles poursuivent des études dans des domaines conduisant majoritairement à des professions dites traditionnellement féminines tels le secrétariat $(90 \%)$, les sciences infirmières $(75,5 \%)$, le droit et l'administration (68\%), commerce et comptabilité $(63,6 \%)$, la normale $(59 \%)$, alors qu'elles représentent moins de $25 \%$ dans les sections scientifiques telles Maths-physique et Biochimie; ce qui limite considérablement 
leur accessibilité aux études supérieures et à des postes de prise de décisions (Gatali, 2000; Gouvernement de la République Rwandaise et UNICEF, 2000).

Il existe très peu de données statistiques sur le nombre de filles/femmes inscrites aux études post-secondaires. Toutefois, il semblerait que le nombre de garçons/hommes par rapport à celui des filles/femmes serait au moins dans l'ordre de 2 pour 1 (PNUD, 2003: 9). En 2001, les femmes constituaient $30 \%$ des 5000 étudiants du campus principal de l'Université Nationale du Rwanda (UNR) sis dans la ville de Butare, et beaucoup parmi elles bénéficiaient d'une bourse d'études (CISCO Learning Institute, 2001: 7). D'autres données existantes (Gatali, 2000: 26) indiquent que les filles représentaient $25 \%$ de l'effectif total des étudiants au supérieur en 1996. Et en prenant comme référence les années 1998 et 1999, sur un total de 5.773 étudiants de l'Université et des instituts supérieurs, 1.502 sont des filles, soit $26 \%$ du total en 1998, alors que leur pourcentage s'élevait à 30\% en 1999.

De plus, malgré leur faible nombre à 'UNR (Gatali, 2000), il se dégage des données disponibles que ces filles ont tendance à fréquenter des disciplines dites traditionnelles. Leur sous représentation pour l'année 1996-1997 est beaucoup plus prononcée dans les facultés d'agronomie $(22 \%)$, de médecine $(17 \%)$ et surtout des sciences appliquées $(3 \%)$ alors que leur pourcentage monte en flèche en Santé publique (41\%) et en sciences de l'éducation (34\%) (Gatali, 2000: 7). Il ne s'agit que d'une continuité de la tendance observée plus haut au niveau secondaire. Les données de 1999-2000 (Gatali, 2000:28) indiquent que les filles constituent $23,3 \%$ de l'effectif total des étudiants de l'UNR. Si on s'en tenait aux données d'avant le génocide de $1994,8,2 \%$ en 1980 et $21,5 \%$ en 1990-1991, nous serions en droit de nous attendre à une représentation des filles avoisinant les $56 \%$ de l'effectif total, soit une augmentation de plus de $200 \%$. N'est-il pas regrettable de constater que sur une période de dix ans, le pourcentage des filles au supérieur soit passé de $21,5 \%$ à $23,3 \%$ soit une augmentation de $1 \%$ ? Pourtant, en rendant l'école obligatoire en 1962, on envisageait instruire un grand nombre de Rwandais sans distinction de sexe. Malheureusement les données rapportées sont loin des résultats escomptés: les femmes sont minoritaires à tous les niveaux d'enseignement et la plupart poursuivent des études dans des domaines ou disciplines conduisant à des emplois peu valorisants et moins rémunérés (OIT, 1984; Gouvernement de la République Rwandaise et UNICEF, 2000). Mais comment expliquer ces réalités?

Malgré l'accent mis sur l'éducation supérieure, seulement $2 \%$ de la population cible en bénéficie alors que le gouvernement y investit $70 \%$ du budget total alloué à l'éducation (World Bank, 2003 :3). Toutefois, les inégalités de genre, régionales et socio-économiques sont les plus manifestes au niveau de l'enseignement supérieur. 


\section{Renforcer les pouvoirs des filles pour atteindre l'équité de genre en éducation}

Le Ministère du Genre et de la Promotion de la Femme et les organisations des femmes jouent un rôle crucial dans la promotion de l'empowerment des filles et l'équité de genre en éducation. Le Ministère du Genre et de la Promotion de la Femme qui a vu le jour en 1999, après diffusion du Ministère du Genre, de la Famille et des Affaires Sociales dont les débuts remontent de 1992, a dans ses attributions le statut des femmes et travaille avec et appuie les organisations des femmes. Son mandat consiste à promouvoir l'égalité entre les femmes et les hommes comme une clé essentielle à la réconciliation, la reconstruction et le développement du pays :

Dans son souci de promouvoir un développement participatif et durable, le Gouvernement de l'Unité Nationale a opté parmi les multiples stratégies visant à pallier aux problèmes structurels et ceux conséquents au génocide, la promotion de l'égalité des genres et l'intégration de la femme au développement. (République Rwandaise, Ministère du Genre et de la Promotion de la Femme, 1999: 3).

Pour ce faire, 12 secteurs prioritaires ont été désignés au sein de ce ministère en vue de la participation équitable des femmes au développement national. Il s'agit de : pauvreté, éducation et formation, santé, VIH et SIDA, violence contre les femmes, économie, l'environnement et le contrôle des ressources naturelles, les jeunes filles, les mécanismes institutionnels pour la promotion de l'égalité de genre, la prise de décision, les droits de l'homme et des femmes, la paix et les femmes, et les médias.

Une des priorités de ce ministère (République Rwandaise, MIGEPROFE, $1999: 10$ ) est « de s'impliquer dans des programmes qui militent pour l'autodétermination des femmes et les assiste pour assumer leurs rôles dans le développement». Un des domaines majeurs de ses activités a été la sensibilisation aux questions d'équité de genre. Avec l'appui de l'UNIFEM, «a Gender Desk » a été mis sur pied au Parlement et la ministre de MIGEPROFE a animé des ateliers auprès des responsables gouvernementaux dont entre autres les fonctionnaires, les membres du parlement, les ministres et les secrétaires généraux. L'objectif du «Gender Desk» et des ateliers de sensibilisation aux questions du genre était de définir le concept genre dans un contexte rwandais, de promouvoir les droits des femmes et enfin d'arriver à des stratégies susceptibles de s'attaquer aux inégalités de genre par le biais des réformes juridiques et du développement du pays, comme l'a fait remarqué the Women's Commission for Refugee Women and Children $(2000: 6)$. Toutefois, il s'agit d'un défi de taille comme d'ailleurs le soulignait Letitia Kayisire, Responsable du Programme Genre au UNDP, cité dans Women's Commission for Refugee Women and Children $(2000: 6)$

In 1995-1996, the word gender meant (to men), Ah, you want to take our place. We needed to find other ways to say it. So we use the word ukuzuzanya which means complementarity rather than uburinganire or equality now. 
[En 1995-1996, le terme genre signifiait (pour les hommes), Ah, vous voulez prendre notre place. Nous avions besoin de trouver d'autres façons de le dire. Alors, nous utilisons le vocable ukuzuzanya qui signifie complémentarité plutôt que uburinganire ou égalité maintenant.] (Traduction libre).

Sensibiliser à la problématique de genre fait partie de l'approche utilisée par le Réseau des Femmes qui a fait de l'éducation des filles une de ses priorités. Au chapitre de l'éducation de la jeune fille, malgré que les responsables de Réseau ont de quoi se réjouir, elles avouent que la bataille sera de longue haleine. En effet, grâce aux recettes des activités microfinances menées par les femmes membres avec l'aide financière du Réseau " ces femmes réussissent à envoyer leurs enfants à l'école». Malgré les rôles et responsabilités qu'assume la femme rwandaise qui autrefois revenaient à l'homme, la fille n'a pas plus droit à la scolarisation qu'avant la guerre et le génocide de 1994. Et c'est là une préoccupation majeure des responsables de Réseau de femmes et qu'elles mettent au cœur de leurs activités de formation :

la mère rwandaise comprend maintenant que la fille doit aussi fréquenter l'école et que sa formation est aussi importante que celle du garçon mais à cause de la pauvreté, quand il y a des choix à faire, c'est encore la fille qu'on retire de l'école. On suppose que pour ce qui est des travaux domestiques, elle sait déjà quoi faire comparativement à son frère. Notre défi maintenant est de former les parents pour qu'ils préparent autant les garçons et les filles à s'impliquer dans des travaux domestiques de façon à ce que ce ne soit pas toujours la fille qui interrompe ses études lorsque la famille fait face à des difficultés financières. [Extrait d'entrevue]

Une autre des contributions majeures de cette Organisation en terme de l'éducation de la jeune fille est d'avoir plaidé en faveur des jeunes filles qui tombent enceintes en cours de scolarité. Bien que la grossesse précoce ou non désirée reste encore un facteur important de déperdition des filles, avant la guerre et le génocide de 1994, une fille enceinte se voyait automatiquement expulsée de l'école.

Avec la guerre et le génocide, la grossesse chez les jeunes filles est devenue un fléau à cause surtout du viol, mais aussi les jeunes orphelines et pauvres succombent facilement à des tentations matérielles. Aujourd'hui, il y a plus de tolérance dans les écoles en cas de grossesse en autant que la jeune fille arrive à surmonter le complexe qui va avec et réussisse à avoir les moyens de poursuivre ses études. [Extrait d'entrevue]

Le Réseau a donc joué un rôle important afin que la mesure punitive, qui consistait à renvoyer la jeune étudiante dans pareille situation, soit levée. Il est courant aujourd'hui de retrouver dans plusieurs établissements de jeunes filles mères qui accueillent leurs enfants aux heures de pause pour les faire téter. Mais le Réseau soutient que le gouvernement devrait faire mieux pour ne pas laisser ces filles à elles mêmes. En collaboration avec d'autres organisations de 
femmes comme FAWE-Rwanda, Pro-Femmes/Twese Hamwe, Club Mamans Sportives, S.O.S Ramira, GIRIBAMBE ...Le Réseau continue à se battre "pour que la fille ne soit pas la seule responsable face à sa grossesse non désirée et reçoive une protection surtout que dans la plupart des cas les auteurs de ces grossesses sont les enseignants de ces fille." [Extrait d'entrevue]

Malgré la fierté qu'elles affichent (à juste titre) de leur succès quant à leur contribution à l'amélioration de la situation de la femme/fille rwandaise qu'elles résument par: kubanza kumva ikibazo cya Gender :comprendre d'abord la problématique de genre; warangiza ukareba ukuntu wacyumvisha undi mugore, ukuntu kimeze n'ingaruka mbi kimufiteho: chercher ensuite à l'expliquer à une autre femme ainsi que les conséquences des inégalités de genre sur elle; mwarangiza mugashaka ingamba zo kukirwanya: chercher ensemble à endiguer ces conséquences; mukaba abavugizi b'abandi, mu bantu no mu nzego zishinzwe ibyo bibazo: être des porte-parole des autres femmes dans la communauté et auprès des instances décisionnelles; no gukomeza kuba ijisho rya rubanda rugufi rireba kure mu birebana n'ibabazo by'abagore n'amajyambere yabo nay'igihugu: continuer à être le salut de la masse rurale relativement à cette problématique.

Les responsables reconnaissent que de nombreux problèmes persistent et c'est pourquoi l'éducation de la jeune fille, le SIDA, les femmes dans le processus de décision, la pauvreté et la pacification restent toujours des aspects focaux de leur plan d'avenir "en ayant toujours comme toile de fond la perspective genre," clament-elles avec conviction.

En plus de sensibiliser aux questions de genre, le MIGEPROFE s'implique et supporte des initiatives entreprises par les organisations des femmes pour promouvoir l'accès des filles à l'éducation. Pro-femmes/Twese Hamwe est le parapluie de toutes les organisations rwandaises de femmes et défend activement, et ce à tous les niveaux, l'accès des femmes à des services de base comme l'éducation et la promotion des droits des femmes (CISCO Learning Institute, $2001: 6$ ). Pour répondre aux préoccupations soulevées par le problème d'analphabétisme chez les femmes rwandaises la plupart des organisations de femmes ont concentré leurs efforts sur l'éducation des filles. En mars 1999, FAWE, Pro-femmes/Twese Hamwe et MIGEPROFE ont organisé une série d'ateliers et des programmes media de sensibilisation sur l'importance de l'éducation des filles (Newbury \& Baldwin, 2000b :7). Une initiative similaire, mise sur pied en 1999 par Réseau des femmes et FAWE en collaboration avec l'association des femmes étudiantes à l'UNR consistait à lancer une étude visant à déterminer les raisons d'abandon scolaire chez les filles et à développer un programme d'action afin de sensibiliser les filles et leurs parents sur l'importance de la poursuite des études par les filles (Newbury \& Baldwin, 2000b :7).

Dans son effort d'améliorer l'accès des filles défavorisées aux études secondaires, le Forum for African Women Educationalists pour le Rwanda, appuie l'École secondaire FAWE des filles au Rwanda, une école secondaire uniquement pour filles et avec internat. Cette école a déjà octroyé 32 bourses 
d'études et a formé jusqu'à 320 filles. FAWE a également fourni un équipement scientifique, des fournitures et des manuels de cours et a ouvert un centre d'ordinateurs pour encourager l'accès aux nouvelles technologies (CISCO Learning Institute, 2001: 6). Cette organisation a mis sur pied plusieurs autres activités à l'endroit des filles et en particulier des filles orphelines les invitant à parler davantage de leurs problèmes. FAWE $(2002: 4)$ reporte que certaines de ces activités permettent aux filles de développer un intérêt pour les maths et les sciences longtemps considérés comme des domaines réservés aux garçons.

Avec un appui financier de RWI, le Club Mamans Sportives (CMS), une organisation des femmes rwandaises créée en exile avec l'objectif de promouvoir le rôle des femmes dans le développement, a mis sur pied un premier centre pour femmes dont la principale activité est d'apprendre à lire et écrire aux filles ainsi que de leur apprendre un métier (Women's Commission for Refugee and Children, 2001: 10). En vue de démanteler les barrières de genre et d'améliorer les conditions de vie des orphelines, l'Association des Guides du Rwanda (AGR) apprend à coudre aux jeunes filles orphelines et les initient à des projets générateurs de revenu basés sur la couture (Women's Commission for Refugee and Children, 2001: 21). Aujourd'hui beaucoup de ces jeunes filles qui ont complété la formation sont propriétaires des salons de couture avec l'appui de ARG dont l'approche proactive de sensibilisation aux questions de genre a permis aux parents et à leurs filles de comprendre l'importance pour les filles de ne pas quitter l'école sans compléter la formation (Women's Commission for Refugee and Children, 2001:10).

\section{Conclusion: la longue route vers uburinganire ou «égalité maintenant »}

Malgré que le Rwanda a fait quelques progrès impressionnants dans le domaine de l'éducation, une analyse socio sexuée révèle que l'accès des filles à l'éducation est encore sujet à d'innombrables inégalités de genre, socioéconomiques et de disparités régionales: l'équité de genre en matière d'éducation est loin d'être atteinte. Comme la Banque Mondiale (World Bank, 2003: 8) le fait si bien remarquer, il faudra aller au delà des résultats déjà atteints selon lesquels des disparités socioéconomiques s'estompent parmi les élèves qui fréquentent l'école primaire. On pourrait en faire encore plus afin de permettre aux enfants les plus vulnérables d'aller à l'école, dont entre autres les doubles orphelins, les enfants de la rue, les enfants des zones rurales ainsi que ceux qui proviennent des familles les plus pauvres. Et au sein de ces différents groupes, une plus grande priorité doit être accordée aux filles afin d'améliorer leur accessibilité aux études secondaires et supérieures tout en inscrivant la situation des filles dans un cadre social, économique et politique plus global, si elles doivent assumer leur rôle de partenaires égales dans le développement; une lutte à laquelle les organisations de femmes se sont adonnées particulièrement après 1994. Comme les responsables de Réseau de Femmes nous le disent :

Malgré le nombre élevé de jeunes filles dans la plupart des familles, les mères hésitent encore à donner priorité à la scolarisation de ces filles et continuent à les considérer comme une main d'œuvre appréciable pour 
les travaux domestiques parce qu'elles sont dans le besoin. Si les politiques permettaient d'améliorer les conditions de travail et de vie de la plupart des mères, on pourrait libérer les jeunes filles des tâches familiales et de survie pour qu'elles puissent fréquenter l'école. [Extrait d'entrevue]

En l'absence de remède miracle pour contrer le problème des inégalités de genre, c'est à la femme d'en prendre conscience et de se prendre en charge, l'homme étant appelé à collaborer; une stratégie utilisée par plusieurs organisations de femmes. En remettant en question les rôles traditionnels des filles et garçons afin de permettre à ces dernières d'aller à l'école, les organisations de femmes réussissent en même temps à inculquer une nouvelle vision des rôles des femmes et des hommes, des filles et des garçons dans la société rwandaise.

\section{Notes:}

1. Selon Ship (1994:138) le genre (gender) comme concept évoque «la construction sociale de la masculinité et féminité, des rôles sociaux des hommes et des femmes qui est façonnée par l'histoire, mise en contexte par la culture et est particulière à la classe sociale. Il faut ajouter que la masculinité et la féminité sont aussi racisées dans le contexte occidental.

2. Malgré l'engagement constitutionnel de rendre l'enseignement primaire obligatoire et gratuit, les parents ont dû payer et paient encore des frais d'inscription pour l'école primaire et assurent d'autres dépenses directement liées à la scolarité. Rendre l'éducation primaire universellement accessible a été et reste un poids financier pour l'État (Gouvernement de la République Rwandaise et UNICEF, $2000: 76$ ).

3. Selon l'étude du Gouvernement de la République Rwandaise et UNICEF (2000), $94 \%$ de la population rwandaise vit en zones rurales.

\section{Références}

African Development Bank/Banque africaine de développement. (2002/2003). Gender, poverty and environmental indicators on African countries. Abidjan.

Berger, G. (1990). École en Afrique noire francophone. In Revue française de la pédagogie. Paris: Institut pédagogique national.

CIDA. (2002). Rwanda programming framework. Framework for Canada's program of development cooperation with Rwanda. Ottawa. http://www.acdicida/CIDAWEB last updated 2002-12-30. Consulted 1/16/2003

Coquery-Vidrovitch, C. (1994). Les africaines: Histoire des femmes d'Afrique Noire du XIXième au XXième siècle. Paris: Éditions Desjonquères.

Dagenais, H. (1994). Méthodologie féministe pour les femmes et le développement. In M.F. Labrecque (dir), L'équité devant soi: sexes, rapports sociaux et développement international, p.258-280. Ottawa:CRDI. 
Djossou-Segla, A. \& Mujawamariya, D. (1998). Le cadre macro politique et culturel de la socialisation des femmes en Afrique: les cas yorouba et fon du Bénin. Revue d'Éducation Comparée et des Relations Internationales en Afrique, 1 (2), 41-67.

FAWE. (2002).Girls' Empowerment FAWE Tuseme Clubs in Rwanda. http://www.fawe.org/FAWE5thGA2002/Document/GA5GirlsEmpowermentRwa nda.pdf

Gatali, J-B. (2000). Recherche sur «les raisons de la non scolarisation des filles au Rwanda. Kigali: Pro-Femmes/Twese Hamwe \& Fonds des Nations Unies pour la Population.

Gouvernement de la République Rwandaise \& UNICEF. (2000). Situation des enfants et des femmes du Rwanda: Survie, développement et protection. Kigali.

Hamilton, H. (2000). Rwanda's women: The key to reconstruction. The Journal of Humanitarian Assistance. http://www.jha.ac/greatlakes/b001.htm Document posted: 10 May 2000.

Jefremovas, V. (1992). Loose women, virtuous wives and timid virgins: Gender and the control of resources in Rwanda. Canadian Journal of African Studies 25 (3), 378-395.

Kumar, K. (2001). Aftermath: Women and women's organizations in post conflict societies: The role of international assistance. Washington: USAID Program and Operations Report No. 28.

Labourie-Racapé, A. (1996). La quatrième conférence mondiale sur les femmes: priorités et enjeux des programmes régionaux. In Thérèse Locoh et al. (Eds.). Genre et développement: des pistes à suivre. Paris: EHESS-INED-INSEE-ORSTOMUniversité Paris VI, Documents et manuels de CEPED 5, 77-95.

Mujawamariya, D. (1992). La femme africaine dans son double rôle d'épouse et de mère», Présentation au Colloque sur l'Émancipation de la Femme Africaine et les Problèmes de son Intégration dans la Société Québécoise Moderne, 21 mars 1992, à l'Université Laval.

Mukakayumba, E. (1995). Rwanda: la violence faite aux femmes en contexte de conflit armé généralisé. Recherches Féministes 8 (1), 145-154.

Newbury, C. \& Baldwin, H. (2000a). Aftermath: Women in post genocide Rwanda. Washington: USAID, Center for Development Information and Evaluation, Working paper No. 303 July 2000.

(2000b). Aftermath: women's organizations in post conflict Rwanda. Washington: USAID, Center for Development Information and Evaluation, Working paper No. 304 July 2000.

O.I.T. (1984). Le défi de l'emploi pour le Rwanda: problématique structurelle en stratégies proposées en vue du IIIe Plan quinquennal de développement, 19821986: rapport de la mission pluridisciplinaire pour une étude d'ensemble des problèmes d'emploi au Rwanda, janvier-février 1981; rédaction finale actualisée, 1983-1984, $1^{\text {ère }}$ édition. Addis-Abeba: B.I.T, Programme des emplois et des compétences techniques pour l'Afrique.

Okojie, C. (2001). Female under-schooling in Africa as assessed by the African Academy of Sciences Research Program. Colloque international Genre, population et développement en Afrique, Abidjan, 16-21 juillet 2001; unpublished paper.

Patemostre, B. (1983). Le Rwanda et son effort de développement: Antécédents historiques et conquêtes de la révolution rwandaise.

PNUD (2002). Rapport mondial sur le développement humain. New York.

Rathgeber, E. (1994). WID, WAD, GAD: Tendances de la recherche et de la pratique dans le champs de développement. In H. Dagenais \& D. Piché (Eds.), Women, feminism and developmentffemmes: Féminisme et développement (pp.77-93). Montréal: McGill-Queen's University Press. 
République Rwandaise, Ministère du genre et de la promotion de la femme (1999). Politique sectorielle. Kigali.

Ristock, J. \& Pennell, J. (1996). Community research as empowerment: Feminist links, postmodern interruptions. Toronto: Oxford University Press.

Siaens, C.S. \& Wodon, Q. (2004). Are orphans vulnerable? Evidence from Rwanda.

http://www.worldbank.org/sp/safetynets/PDFfiles/are\%20orphans \%20vulnerable.pdf

Ship, S.J. (1994). "And what about gender?" Feminism and international relations' theory's third debate. In Claire S. and W. Cox (Eds.), Beyond positivism. Critical reflections in international relations (pp. 129-151). Boulder: Lynne Rienner,

UNAIDS. (2002). Rwanda: Epidemiological fact sheets on HIV/AIDS and sexually transmitted infections 2002 Update. www.aids.africa.com consulté en juillet 2005.

UNICEF. (1997). Children and women of Rwanda: A situation analysis of social sectors. Geneva

------- (2002). UNICEF Statistics Rwanda. http:www.unicef.org/stas/Country_1 Page14

U.N.R. (1978). Éléments d'histoire du Rwanda. In Études rwandaises, vol XI, numéro spécial de mars.

Uwimana, C. (1992). Les inégalités de chances entre les hommes et les femmes en éducation au Rwanda depuis 1962. Essai présenté pour l'obtention du grade de maître ès en arts. École des gradués, Université Laval.

Women's Commission for refugee women and children (1997). Rwanda's women and children: The long road to reconciliation. New York and USAID Women in Transition Office Kigali, (2000). Rebuilding Rwanda: "A struggle men cannot do alone" Delegation Report. Winter 2000. http: www.womenscommission.org/pdf/rw.pdf

- (2001). You cannot dance if you cannot stand: A review of the Rwanda women's initiative and the United Nations High Commission for Refugees commitment to gender equity in postconflict societies 2001. New York and USAID Women in Transition Office Kigali.

World Bank. (1998). Rwanda poverty note: Rebuilding an equitable society: Poverty and poverty reduction after the Genocide. Washington: World Bank Report No. 17792-RW).

(2003). Education in Rwanda: Rebalancing resources to accelerate post-conflict development and poverty reduction. Executive Summary. Washington.

http://worldbank.org/publications/pdfs/15610frontmat.pdf 